\title{
Fractura esternal: descripción de tratamiento quirúrgico
}

\author{
Patricio Salas V. ${ }^{1}$, Felipe Bannura Y. ${ }^{1}$, María Eliana Solovera R. ${ }^{1}$ y Andrea Ramírez B. ${ }^{1}$
}

'División de Cirugía Facultad de Medicina Pontificia Universidad Católica de Chile. Santiago, Chile.

Recibido 2020-09-29 aceptado 2020-11-30

Correspondencia a: Dr. Patricio Salas V. psalas@med.puc.c

\section{Sternal fracture: surgical management description}

Aim: To describe demographic characteristics and surgical treatment carried out on patients with a sternal fracture (SF) in the last 5 years. Materials and Method: Retrospective descriptive study of patients operated on for SF between January 2015 and January 2020. We analyzed age, sex, morbid history, hemodynamics on admission, causal mechanism and characteristics of sternal injury, associated injuries, surgical indication and complications. Results: During the period, 9 patients were admitted to our hospital ( 7 men) from 21 to 91 years old. All were operated. Most were admitted with stable hemodynamics. The mechanism was always traumatic. The surgical indications were: intractable pain, alteration of ventilatory mechanics, flail chest, deformity and aid in the rehabilitation of spinal cord trauma. Discussion: SF is an infrequent pathology, its surgical resolution reported worldwide being even scarce. Conclusions: We present the first report of a series of cases of SF operated in Chile. Sternal osteosynthesis allows the management of EF with good functional results with a low morbidity rate. The results obtained are comparable to those observed in the international literature.

Key words: sternal fracture; thoracic Injuries; osteosynthesis.

\section{Resumen}

Objetivo: Describir características demográficas y tratamiento quirúrgico realizado a pacientes con fractura de esternón (FE) en los últimos 5 años. Materiales y Método: Estudio descriptivo retrospectivo de pacientes operados por fractura esternal entre enero de 2015 y enero de 2020. Se analizaron edad, sexo, antecedentes mórbidos, hemodinamia de ingreso, mecanismo causal, características de lesión esternal, lesiones asociadas, indicación quirúrgica y complicaciones. Resultados: Durante el período ingresaron a nuestro hospital 9 pacientes ( 7 hombres) de 21 a 91 años. Todos fueron operados. La mayoría ingresó con hemodinamia estable. El mecanismo fue siempre traumático. Las indicaciones quirúrgicas fueron: dolor intratable, alteración de la mecánica ventilatoria, tórax volante, deformidad y ayuda en la rehabilitación de un trauma raquimedular. Discusión: La FE es una patología infrecuente, siendo aún más escasa su resolución quirúrgica reportada a nivel mundial. Conclusiones: Presentamos el primer reporte de una serie de casos de FE operada en Chile. La osteosíntesis esternal permite el manejo de la FE con buenos resultados funcionales con baja tasa de morbilidad. Los resultados obtenidos son comparables a los observados en la literatura internacional.

Palabras clave: fractura esternal; lesiones torácicas; osteosíntesis.

\section{Introducción}

La fractura esternal (FE) es una patología infrecuente a nivel mundial y en Chile representa solo el $2,9 \%$ de los ingresos por traumatismo torácico ${ }^{1}$. Por lo anterior, existe escasa literatura que documente y actualice las características de la FE y el manejo quirúrgico.

La FE está asociada a traumatismos de alta energía con impacto directo sobre la pared anterior del tórax. También está descrito el mecanismo de flexo-extensión forzada ("latigazo") como causal de luxación manubrio-esternal eventualmente asociada a lesión de columna vertebral. La presentación de FE aislada es poco frecuente y suele acompañarse de otras lesiones, siendo esto la principal causa de morbimortalidad.

El diagnóstico es radiológico, siendo el gold 
standard en el paciente con trauma de alta energía la tomografía computada de tórax con contraste endovenoso y eventualmente fase angiográfica, con el fin de descartar lesiones de importancia asociadas. La radiografía de esternón anteroposterior y principalmente la lateral es un elemento de apoyo importante, de menor sensibilidad y especificidad.

El manejo es médico hasta en el 95\% de los ca$\operatorname{sos}^{2-4}$, reservando la cirugía para casos excepcionales. No hay guías de tratamiento publicadas.

\section{Objetivo}

Describir las características y tratamiento quirúrgico de pacientes con $\mathrm{FE}$ tratados con osteosíntesis esternal en nuestro hospital en un período de 5 años.

\section{Materiales y Método}

Estudio descriptivo retrospectivo de pacientes operados por FE en el hospital por el equipo de cirugía de tórax, entre enero de 2015 y enero de 2020. La información se obtuvo a partir de fichas clínicas. Se ordenaron los datos mediante planilla Excel $^{\circledR}$ y el programa SSPS 21. Se describen las siguientes va- riables: edad, sexo, antecedentes mórbidos, hemodinamia de ingreso, mecanismo causal, características de la lesión esternal, lesiones asociadas, decisión de cirugía, tratamiento quirúrgico realizado y complicaciones posoperatorias.

La técnica quirúrgica utilizada fue la siguiente: por abordaje medio esternal se redujo el foco de fractura. Se midió el grosor del esternón para definir longitud de tornillo a usar. Luego se decide el tipo y número de placas de osteosíntesis. Esto dependerá del rasgo de fractura, prefiriendo generalmente más de una placa para dar más estabilidad a la reparación. Para reparar un rasgo oblicuo las placas se colocaron a distinto nivel. Los casos hasta 2018 fueron reparados con el sistema matrix (DePuySynthes ${ }^{\odot}$ ) y desde esa fecha usamos sistema Sternalock Blu $\left(\right.$ Zimmerbiomet $\left.^{\odot}\right)$. Una vez reducida la fractura o luxación se fijaron las placas con los tornillos escogidos. Luego se afrontaron los pectorales y se completa el cierre por planos (Figura 1). En solo a un tercio de los pacientes se dejó drenaje submuscular por amplia disección. Las osteosíntesis de fracturas costales asociadas fueron en dos pacientes, uno con fracturas costales del arco anterior derecho desde la tercera a quinta costilla y el segundo caso con fracturas costales de la primera costilla bilateral asociado a fracturas de la segunda a la octava costilla derecha en su arco lateral y de la segunda a

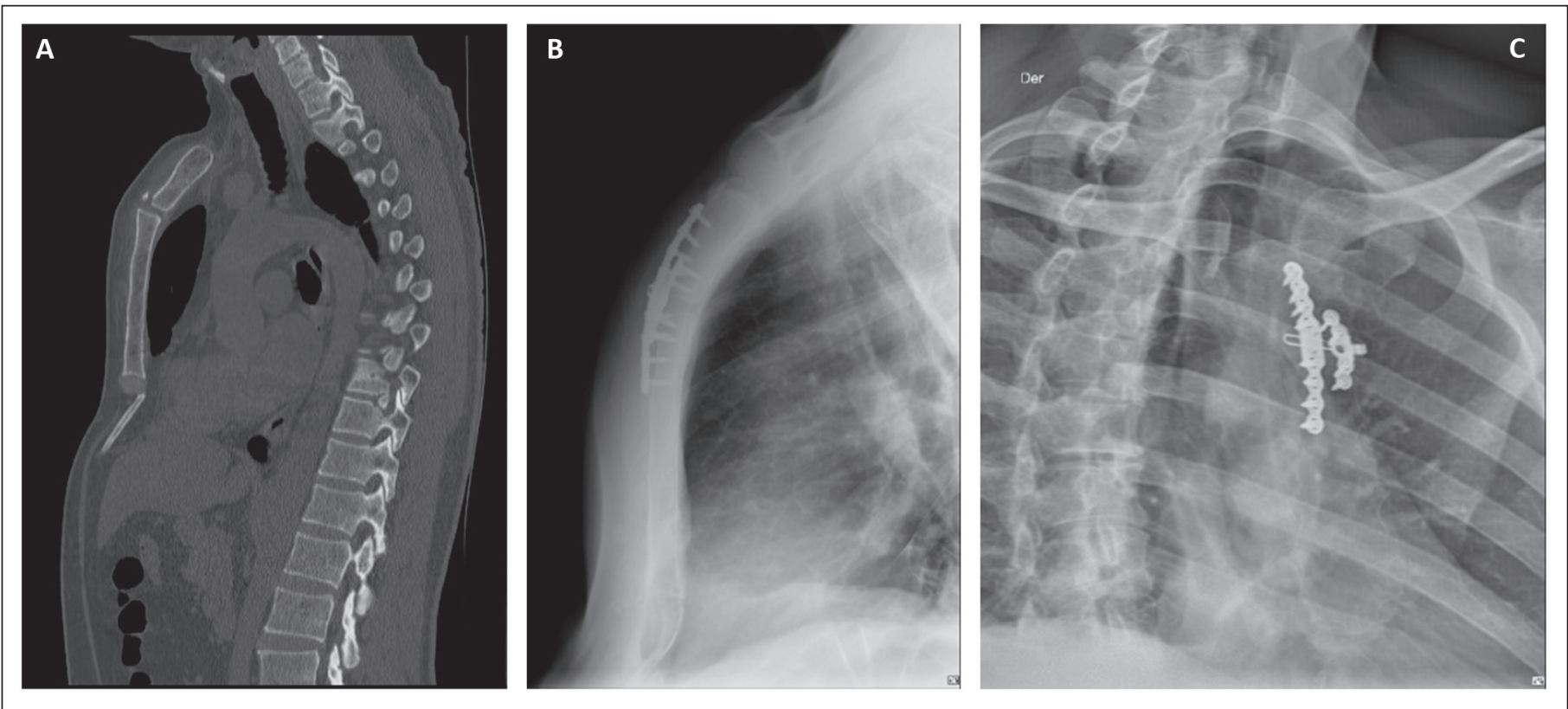

Figura 1. Hombre 63 años. A: TAC de tórax, corte sagital, se aprecia luxofractura de articulación manubrio-esternal con desplazamiento hacia posterior del manubrio. B: Radiografía de esternón lateral posoperatoria. Se aprecia correcta alineación del esternón por material de osteosíntesis (2 placas). C: Radiografía de esternón AP posoperatoria. Muestra 2 placas de osteosíntesis. 


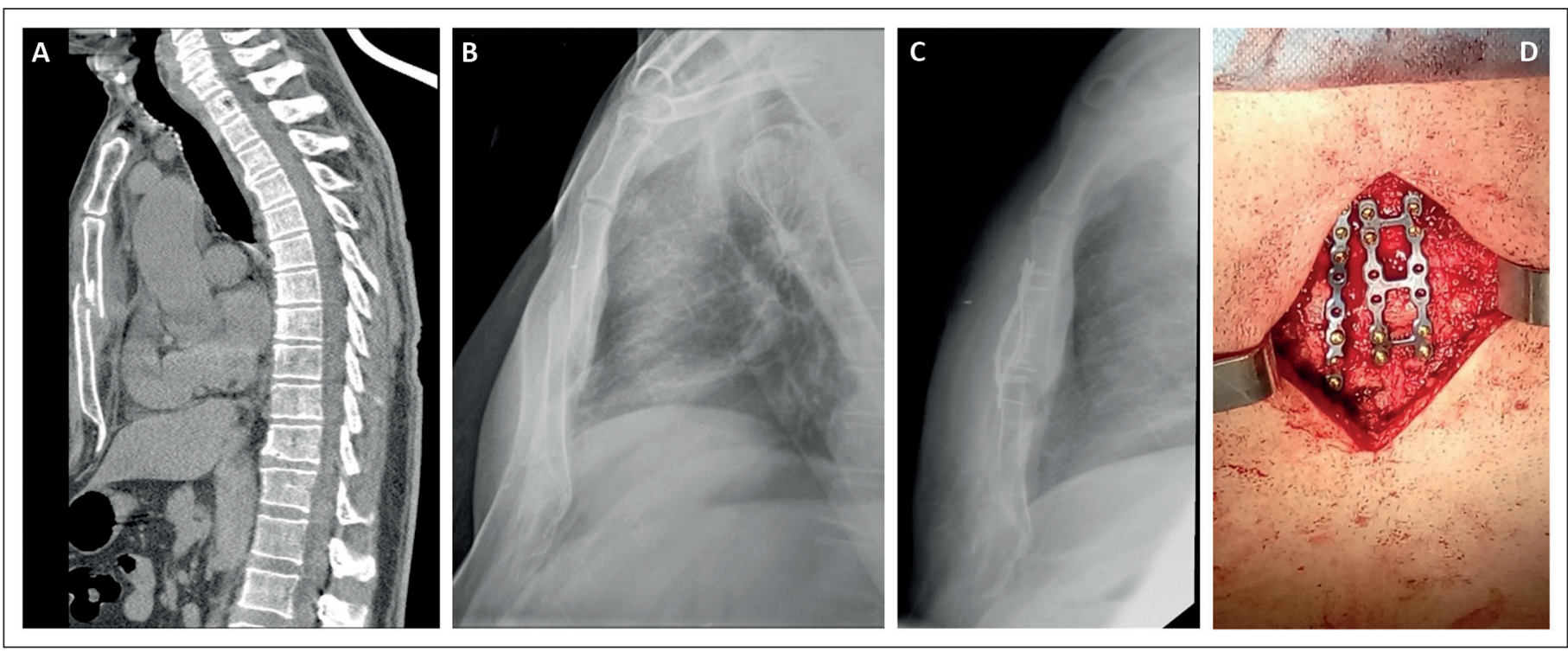

Figura 2. Hombre de 65 años. A: TAC de tórax corte sagital. Se aprecia fractura de rasgo completo del tercio medio del cuerpo esternal. El paciente presentaba poco dolor, no había movilidad de los segmentos óseos y la fractura estaba alineada. B: Radiografía lateral de esternón. Tomada a las 3 semanas del accidente inicial. Muestra fragmentos óseos cabalgados. C: Radiografía lateral de esternón. Muestra foco de fractura reducido y alineado con 2 placas de osteosíntesis. D: Fotografía intraoperatoria muestra rasgo de fractura reducido y fijo con 2 placas de osteosíntesis.

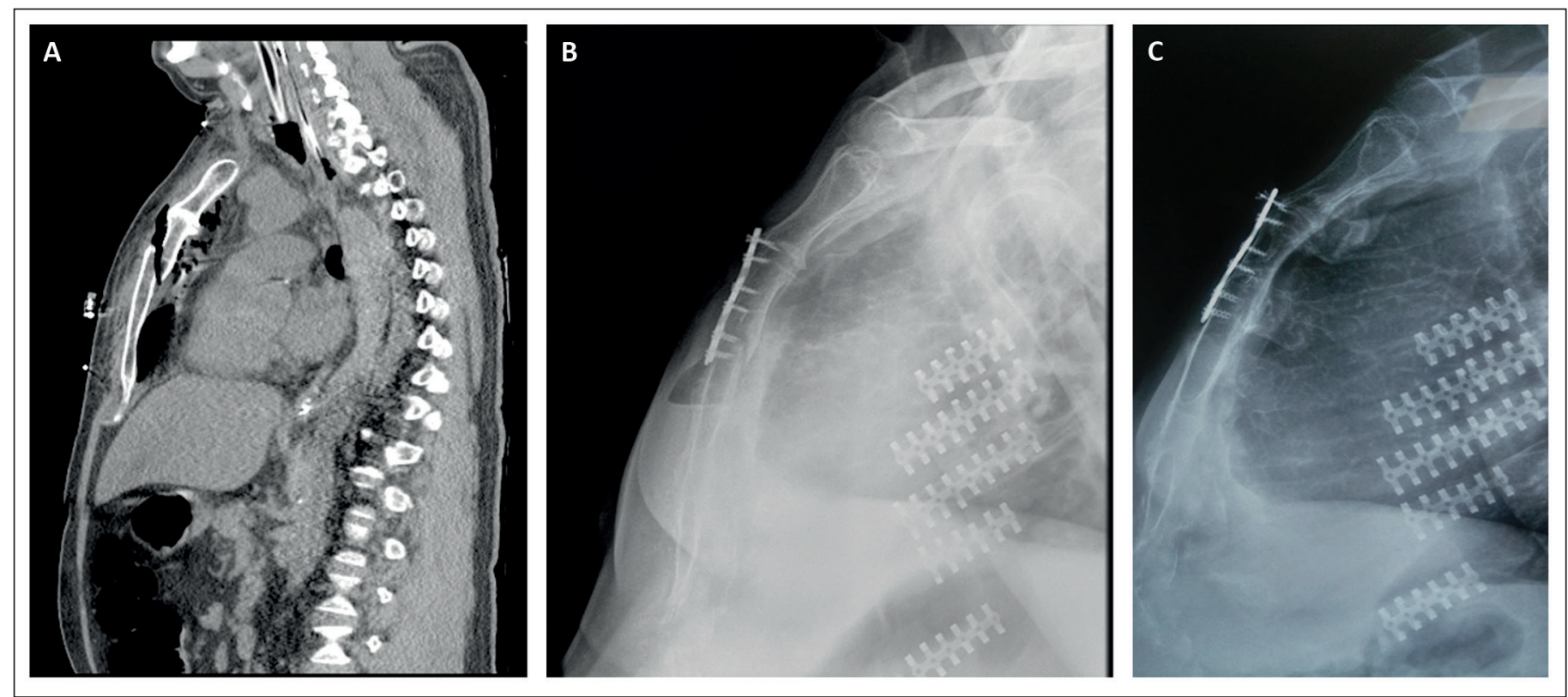

Figura 3. Hombre 63 años. A: TAC de tórax sagital. Se observa fractura de rasgo oblicuo, desplazada, de tercio medio del cuerpo del esternón. B: Radiografía lateral de esternón. 3 semanas posoperatoria. Placas de osteosíntesis esternal y costales in situ. C: Radiografía lateral de esternón. 15 meses posoperatorio. Se aprecia placa de osteosíntesis in situ con esternón alineado y foco de fractura consolidado. También se aprecian placas de osteosíntesis costales derechas.

la séptima costilla izquierda (Figura 3). En ambos pacientes la osteosíntesis costal se realizó por otro abordaje lateral. Se operaron 2 luxaciones esternocondrales que se repararon suturando el cartílago costal al esternón con prolene 0 .

\section{Resultados}

De los 9 pacientes con FE, todos fueron operados. Son 7 hombres. La mediana de edad fue 47 años, con rango entre 21 y 91 años (Tabla 1). No 
hubo compromiso hemodinámico en 7 pacientes. En cuanto al mecanismo, todos presentaron un traumatismo, en 6 fue por accidente vehicular, 2 caídas de altura y 1 contusión torácica directa. Todos los pacientes presentaron al menos una lesión asociada, siendo lo más frecuente fracturas costales, seguido por hematoma retroesternal, hemo o neumotórax, lesiones osteoarticulares, lesiones neurológicas y contusión pulmonar (Tabla 2). En cuanto a los pacientes con fracturas costales asociadas, la mayoría de ellas $(n=8)$ son unilaterales. Hubo tres pacientes con fractura costal única, mientras que los demás pacientes presentaron 3, 4 y 15 fracturas costales. En 2 pacientes se realizó osteosíntesis costal (Tabla $3)$. En cuanto a la lesión esternal, 7 pacientes presentaron FE, mientras que en 2 pacientes se objetivó luxo fractura de la articulación manubrio-esternal. Del subgrupo FE, una de ellas fue en el manubrio y las restantes a nivel del cuerpo esternal. De las fracturas a nivel del cuerpo esternal, 3 fueron a nivel del tercio distal, 3 en el tercio proximal y 2 en tercio medio. Dos pacientes presentaron más de un rasgo de fractura esternal. En cuanto a los rasgos de fracturas, se encontró rasgo oblicuo en 4 pacientes, transverso en 2 pacientes, longitudinal en uno y conminuta en un paciente.

Las indicaciones quirúrgicas se describen en la Tabla 3.

No se presentaron complicaciones posoperatorias graves, 2 complicaciones Clavien Dindo I: dehiscencia mínima y seroma de herida.

\section{Discusión}

La FE es infrecuente dentro de las enfermedades del tórax, pero ha aumentado su incidencia principalmente por los accidentes de tránsito. Existe escasa documentación en la literatura respecto a la técnica quirúrgica ${ }^{5-11}$, siendo la mayoría casos clínicos aislados. Nuestra serie concuerda con los reportes mundiales en que los más afectados son hombres en $5^{\circ}$ década de vida ${ }^{12}$. Los criterios más usados para definir la cirugía son dolor, mal alineación ósea y no unión. Como muestra la revisión sistemática de Klei ${ }^{12}$ que incluye 191 pacientes, la FE aislada representa un $27 \%$, por lo que debemos ser muy activos en la búsqueda de lesiones asociadas. Los sitios afectados con más frecuencia son la pared torácica (costillas, escápula), órganos torácicos y abdominales, sistema nervioso central y columna vertebral. Llama la atención que no observamos lesiones cardiacas, siendo descartadas con electrocardiograma sin alteraciones agudas y curva de troponinas no elevada. Nuestro manejo consiste en descartar las lesiones asociadas y luego definir la resolución quirúrgica según los criterios de la Tabla 3.

Presentamos una serie heterogénea en cuanto al tipo de lesiones esternales y el tiempo de resolución. Presentamos 2 casos de luxo-fractura manubrio

Tabla 1. Características demográficas de pacientes operados por FE

\begin{tabular}{|ll|}
\hline Características & $\mathbf{n}=\mathbf{9}$ \\
Edad (años) & \\
$\quad$ Promedio & 50 \\
$\quad$ Mediana & 47 \\
$\quad$ Rango & $21-91$ \\
Sexo & \\
$\quad$ Masculino & 7 \\
$\quad$ Femenino & 2 \\
Días desde accidente a reparación quirúrgica & \\
(promedio/rango) & $6,7 / 1$ a 30 \\
Estadía hospitalaria (promedio/rango) días & $16,5(2-107)^{\mathrm{a}}$ \\
Necesidad de VM ( ${ }^{\circ}$ pacientes) & 2 \\
VM (duración en días) & Paciente $1: 2$ días \\
& $\begin{array}{l}\text { Paciente } 2: \text { VM a permanencia } \\
\text { por trauma raquimedular }\end{array}$ \\
\hline
\end{tabular}

aPaciente con trauma medular hospitalizado para rehabilitación. Sin ese paciente el promedio de hospitalización es 5,25 días. VM: ventilación mecánica.

Tabla 2. Lesiones asociadas

\begin{tabular}{|lc|}
\hline & n=9 \\
Fracturas costales & 6 \\
Hematoma retroesternal & 3 \\
Hemo y/o neumotórax & 3 \\
Luxación esterno-condral & 2 \\
Lesiones osteoarticulares "no costales"a & 5 \\
Lesiones neurológicas ${ }^{\mathrm{b}}$ & 4 \\
Contusión pulmonar & 1 \\
\hline
\end{tabular}

${ }^{a}$ Esguince columna cervical, fractura columna vertebral, fractura mandibular. ${ }^{\mathrm{b}}$ Traumatismo encéfalo craneano ( 3 casos), traumatismo raquimedular.

Tabla 3. Motivo de resolución quirúrgica en FE

\begin{tabular}{|lc|}
\hline & $\mathbf{n = 9}$ \\
\hline Dolor intratable & 4 \\
Alteración de mecánica ventilatoria & 4 \\
Mal alineación ósea & 3 \\
Optimizar rehabilitación por trauma raquimedular & 1 \\
\hline
\end{tabular}

Puede existir más de 1 criterio por paciente. 
esternal en que ambos pacientes referían dolor de gran intensidad asociado a inestabilidad. En la Figura 1 observamos un corte sagital de tomografía computada de tórax que muestra una luxación con el manubrio desplazado hacia posterior. El paciente presentaba una clara deformidad y movilidad anormal a nivel del ángulo de Lewis asociado a dolor intenso.

En la Figura 2 se aprecia cabalgamiento de $5 \mathrm{~cm}$ de los bordes óseos, por lo que se decidió la reducción y osteosíntesis con dos placas logrando alineación adecuada. La Figura 3 muestra las imágenes de un hombre de 63 años que sufrió un volcamiento vehicular. Ingresó en ventilación mecánica con diagnóstico de tórax volante. Presentaba un doble rasgo de fractura esternal, oblicuo y longitudinal en el tercio medio. También una luxación externo condral del segundo cartílago costal izquierdo y fracturas costales derechas desde la $3^{\circ}$ a la $8^{\circ}$ por arco lateral y fracturas costales izquierdas de la segunda a la séptima costilla por arco lateral. El esternón se reparó con una placa esternal, logrando adecuada alineación y estabilidad. También se realizó osteosíntesis de fracturas costales derechas. Fue extubado a las $48 \mathrm{~h}$ con tórax estable y presentando poco dolor de las fracturas izquierdas.

El manejo quirúrgico es un procedimiento infrecuente en la resolución de las $\mathrm{FE}^{4}$. La técnica quirúrgica descrita logró estabilidad y consolidación en todos los casos.
La decisión quirúrgica está determinada por factores claros como dolor intratable, alteraciones de la mecánica ventilatoria, deformidad anterior del tórax determinada por mal alineación del rasgo de fractura que además puede producir una falta de consolidación.

\section{Conclusiones}

Existen escasos reportes en la literatura sobre la resolución quirúrgica de las FE, siendo incluso mencionado como controversial en muchos de éstos. Nuestra serie muestra resultados y de morbimortalidad similares a lo reportado a nivel mundial ${ }^{11-13}$.

La resolución quirúrgica es una solución segura y con escasas complicaciones.

\section{Responsabilidades éticas}

Protección de personas y animales. Los autores declaran que para esta investigación no se han realizado experimentos en seres humanos ni en animales.

Confidencialidad de los datos. Los autores declaran que en este artículo no aparecen datos de pacientes.

Conflictos de interés: no hay.

\section{Bibliografía}

1. González LR, Riquelme UA, Fuentes EA, Saldías FR, Reyes MR, Seguel SE, et al. Traumatismo torácico: caracterización de hospitalizaciones durante tres décadas [Thoracic trauma. Experience of three decades]. Rev Med Chile 2018;146:196-205. doi: 10.4067/s003498872018000200196. PMID: 29999156.

2. Ferrer Y, Oquendo P, Morejón Y. Fractura aislada de esternón: Presentación de un caso. Medisur, Cuba 2014;12:889-94.

3. Espinoza R, Aguilera H. Fractura de esternón. Rev Chil Cir. 1999;51:23-8.

4. Athanassiadi K, Gerazounis M, Moustardas M, Metaxas E. Sternal fractures: retrospective analysis of 100 cases. World J Surg. 2002;26:1243-6. doi: 10.1007/s00268-002-6511-5. Epub 2002 Aug 16. PMID: 12181604.

5. Sarquis G, Vejez S, Suizer A,
Reche F. Fracturas traumáticas del esternón: opciones de diagnóstico y tratamiento. Rev Fac Cienc Méd. (Córdoba) 2003;60:13-18.

6. Bonney S, Lenczner E, Harvey EJ Sternal fractures: anterior plating rationale. J Trauma 2004;57:1344-6. 10.1097/01.ta.0000074754.92150.87. PMID: 15625475.

7. Jacinto Tinajero JC, Santillán-Doherty $P$, Argote-Greene LM, Carranza-Martínez I, Duarte-Mancera D, Martín-del-Campo LA, et al. Fracturas traumáticas del esternón: Prioridades y estrategia de manejo. Neumología y Cirugía de Tórax 2009;68:151-5.

8. Castro D, Pacheco R. Manejo quirúrgico de fractura de esternón a propósito de un caso. Rev Inv Inf Salud 2016;11:35-41.

9. Fonseca F. Fractura de esternón. Presentación de un caso. Rev Chil Cir. 2017;69:408-11.
10. Mellado J, Mellado J. Fractura de esternón, fijación con lámina y tornillos. Revisión de la literatura a propósito de un caso. Revista Cubana de Reumatología, 2019;21:70-9.

11. Balén E. Fracturas y luxaciones del esternón. Revista Clínica Española, 1955;27:30-7.

12. Klei DS, de Jong MB, Öner FC, Leenen LPH, van Wessem KJP. Current treatment and outcomes of traumatic sternal fractures-a systematic review. Int Orthop. 2019;43:1455-64. doi: 10.1007/s00264-018-3945-4. Epub 2018 Apr 26. PMID: 29700586; PMCID: PMC6525114.

13. Zhao Y, Yang Y, Gao Z, Wu W, He W, Zhao T. Treatment of traumatic sternal fractures with titanium plate internal fixation: a retrospective study. J Cardiothorac Surg. 2017;12:22. https:// doi.org/10.1186/s13019-017-0580-x 